\title{
Disgeusia: a propósito de um caso clínico
}

Óscar de Barros, ${ }^{1}$ João Carlos Ribeiro, ${ }^{2-3}$ Ângela Ferreira, ${ }^{4}$ Hélder Ferreira, ${ }^{5}$ António Paiva ${ }^{6-7}$

\section{RESUMO}

Introdução: A distorção persistente da sensação gustativa (disgeusia), sendo transitória ou permanente, pode ter influência significativa no quotidiano dos doentes, relacionando-se com perda ponderal, anorexia, desnutrição, quadros depressivos e redução significativa da qualidade de vida. A disgeusia pode ter várias causas, sendo uma delas a cirúrgica, nomeadamente a amigdalectomia. Descrição do caso: Doente do sexo feminino, 38 anos, com história de amigdalite crónica bilateral. Realiza amigdalectomia por lâmina fria e eletrocauterização de pontos hemorrágicos da loca amigdalina, sem complicações cirúrgicas. Inicia quadro de disgeusia caracterizada pela não discriminação dos gostos, referindo que tudo parecia amargo. Na consulta de seguimento hospitalar em otorrinolaringologia (ORL), seis meses após cirurgia, efetuou-se avaliação gustométrica por tiras teste, revelando alterações na discriminação gustativa, referindo o gosto amargo, mesmo quando estimulada com tiras ácidas, doces ou salgadas. A doente iniciou reabilitação gustativa diária, fazendo associação mental com a sensorial. Repetiu-se gustometria um ano após cirurgia. A doente melhorou bastante, mas manteve uma disgeusia seletiva para o amargo na presença de estímulo salgado em baixa concentração.

Comentário: A disgeusia é uma complicação rara da amigdalectomia e de elevada importância para o doente. O procedimento cirúrgico pode atingir os nervos responsáveis pela transmissão sensorial gustativa, direta ou indiretamente, não havendo muitas opções terapêuticas. Estes casos estão subreportados na literatura e os que foram tiveram evolução benigna. A disgeusia pode ser várias etiologias, como higiene oral precária, cirurgia, infeção e tabaco, diabetes, doenças autoimunes, fármacos antimicrobianos, anti-hipertensores e anti-inflamatórios. Nos cuidados de saúde primários, as queixas gustativas são vagas e sem relação patológica aparente, não existindo em Portugal dados acerca da incidência da mesma. É importante sensibilizar os clínicos para esta patologia. Não devemos descuidar a queixa do doente, quer pelo impacto que a mesma tem na qualidade de vida do mesmo, quer pela possibilidade de haver uma patologia de base tratável. É nosso papel avaliar, orientar e tratar.

Palavras-chave: Disgeusia; Paladar; Amigdalectomia.

\section{INTRODUÇÃO}

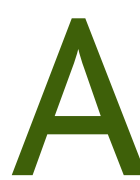
lterações na função gustativa, quer transitórias quer permanentes, podem ter consequências importantes para o quotidiano dos indivíduos, estando relacionadas com alterações ponderais e quadros depressivos. ${ }^{1-2}$ Atualmente são descritos cinco tipos de sensações gustativas: o doce, o amargo, o salgado, o ácido e o umami. A informação gustativa é transmitida superiormente, não só,

\footnotetext{
'Médico Interno de Medicina Geral e Familiar, UCSP Celas, ACES Baixo Mondego 2Médico Assistente Hospitalar, Serviço de Otorrinolaringologia, $\mathrm{CHUC}$ ${ }^{3}$ Assistente Convidado, Faculdade de Medicina da Universidade de Coimbra ${ }^{4}$ Médico Interno de Medicina Geral e Familiar, USF Briosa, ACES Baixo Mondego ${ }^{5}$ Médico Assistente Graduado e Diretor de Serviço, UCSP Celas, ACES Baixo Mondego

${ }^{6}$ Chefe de Serviço, Diretor de Serviço, Serviço de Otorrinolaringologia, CHUC ${ }^{7}$ Professor Catedrático, Faculdade de Medicina da Universidade de Coimbra
}

mas significativamente através do nervo glossofaríngeo.

A amigdalectomia é, na atualidade, a cirurgia mais frequente do foro otorrinolaringológico, nos EUA, Europa e Japão. Está mais frequentemente indicada em doentes com amigdalites de repetição (sete episódios por ano ou cinco episódios em dois anos consecutivos), amigdalite crónica não responsiva ao tratamento médico e abcessos peri-amigdalinos com complicações. Apesar de ser considerada um procedimento de baixa complexidade, tem associados riscos e complicações como qualquer cirurgia. A hemorragia, odinofagia, infeção, danos dentários, otalgia, náuseas e vómitos são os mais comuns. A alteração do gosto, por seu lado, encontra-se entre os menos reportados. Sendo parca a incidência descrita na literatura, as alterações 
gustativas no período pós cirúrgico pode atingir os $8 \%$; no entanto, menos de $1 \%$ permanece a longo prazo. Esta pode ser temporária ou definitiva, parcial (hipogeusia) ou total (ageusia) ou gosto fantasma que está presente na ausência de estímulo gustativo (fantogeusia/alucinações gustativas). ${ }^{3-5}$

A avaliação da alteração gustativa

\begin{tabular}{|c|c|c|c|c|c|c|c|}
\hline \multicolumn{2}{|c|}{$E$} & \multicolumn{2}{|c|}{ D } & \multicolumn{2}{|c|}{ E } & \multicolumn{2}{|c|}{ D } \\
\hline Doce 4 & $\mathrm{X}$ amargo & Ácido 4 & $\mathrm{X}$ amargo & Salgado 2 & $\mathrm{X}$ ácido & Amargo 2 & $\nabla$ \\
\hline Amargo 4 & $\nabla$ & Salgado 4 & $\mathrm{X}$ amargo & Ácido 2 & $\nabla$ & Doce 2 & $\nabla$ \\
\hline Salgado 4 & $X$ amargo & Amargo 4 & $\nabla$ & Doce 2 & $\nabla$ & Ácido 2 & $\nabla$ \\
\hline Ácido 4 & $\nabla$ & Doce 4 & $X$ amargo & Ácido 1 & $\mathrm{X}$ amargo & Controlo & $X$ amargo \\
\hline Ácido 3 & X amargo & Salgado 3 & $X$ amargo & Controlo & $\nabla$ & Salgado 2 & $\mathrm{X}$ amargo \\
\hline Doce 3 & $\nabla$ & Controlo & $\mathrm{X}$ amargo & Salgado 1 & $\mathrm{X}$ amargo & Doce 1 & $\nabla$ \\
\hline Controlo & $\mathrm{X}$ amargo & Amargo 3 & $\nabla$ & Amargo 2 & $\nabla$ & Ácido 1 & $\nabla$ \\
\hline Amargo 3 & $\nabla$ & Doce 3 & $\mathrm{X}$ amargo & Amargo 1 & $\nabla$ & Salgado 1 & $\mathrm{X}$ amargo \\
\hline Salgado 3 & $\mathrm{X}$ amargo & Ácido 3 & $\nabla$ & Doce 1 & $\mathrm{X}$ amargo & Amargo 1 & $\nabla$ \\
\hline
\end{tabular}

Legenda: 1 - Maior concentração; 4 - Menor concentração; E - Lado esquerdo da língua; D - Lado direito da língua. Quando gosto alterado, é descrito qual o gosto identificado após o X.

pode ser feita por vá-

rios métodos, incluindo a eletrogustometria e a gustometria química por tiras teste. ${ }^{6}$

\section{DESCRIÇÃO DO CASO}

Doente do sexo feminino, 38 anos, com história pessoal de $\beta$-talassémia minor, pré-eclampsia com edema cerebral e amigdalite crónica bilateral. Realiza amigdalectomia por lâmina fria e eletrocauterização de pontos hemorrágicos da loca amigdalina. Não foram relatadas complicações cirúrgicas. Duas semanas após cirurgia, na consulta de seguimento pós-cirurgia em ORL, refere alteração do gosto caracterizada pela não discriminação dos gostos, parecendo-lhe tudo amargo.

Seis meses após início do quadro, em nova consulta de seguimento hospitalar, efetuou-se avaliação gustométrica por tiras teste.

A gustometria química por tiras teste baseia-se na aplicação de tiras teste impregnadas com cada um dos quatro gostos (ácido, amargo, doce, salgado) em quatro diferentes concentrações padronizadas. Este protocolo de avaliação não contempla o umami por não terem obtido resultados com a população teste. As tiras são colocadas no lado direito e/ou esquerdo da língua, no terço anterior da língua em extensão, somando um total de 32 testes. Com a língua ainda em extensão, o doente tem de identificar o gosto da tira teste, escolhendo um dos quatro gostos obrigatoriamente. ${ }^{2}$

Aplicando o protocolo de gustometria com tiras tes- te, ${ }^{1}$ obtivemos as alterações descritas no Quadro I.

A doente apresentava alterações na discriminação gustativa, em que para todos os gostos confundia o gosto testado pelo amargo, com predomínio nas tiras de baixa concentração. De notar que em cinco das oito tiras controlo foi referido o gosto amargo. A doente fez reabilitação gustativa, em que diariamente provava os quatro tipos de gosto em causa, fazendo associação mental com a sensorial.

Repetiu-se a avaliação gustométrica 10 meses após cirurgia (Quadro II).

A disgeusia mantém-se, embora seletivamente para o salgado - amargo. Refere ainda que a família se queixa que ela abusa muito do sal quando cozinha, o que vai ao encontro aos resultados encontrados na gustometria, visto só conseguir discriminar o salgado para concentrações elevadas.

Atualmente não há tratamento para a disgeusia secundária a cirurgia. Os casos reportados na literatura observaram uma evolução benigna, sem contudo diferenciarem as várias qualidades de gosto. Esta doente teve uma evolução benigna em menos de dois anos. De facto, comparando este relato de caso com outros semelhantes existentes, verificamos uma melhoria significativa em menos tempo que os habituais dois anos. Este facto pode ser atribuído à reabilitação gustativa diária iniciada em consulta de seguimento em ORL e posteriormente feita pela doente em casa. A doente fez 


\begin{tabular}{|c|c|c|c|c|c|c|c|}
\hline \multicolumn{2}{|c|}{$\mathbf{E}$} & \multicolumn{2}{|c|}{ D } & \multicolumn{2}{|c|}{$E$} & \multicolumn{2}{|c|}{ D } \\
\hline Doce 4 & $\nabla$ & Ácido 4 & $\nabla$ & Salgado 2 & $\mathrm{X}$ amargo & Amargo 2 & $\nabla$ \\
\hline Amargo 4 & $\nabla$ & Salgado 4 & $\nabla$ & Ácido 2 & $\nabla$ & Doce 2 & $\nabla$ \\
\hline Salgado 4 & $\mathrm{X}$ amargo & Amargo 4 & $\nabla$ & Doce 2 & $\nabla$ & Ácido 2 & $\nabla$ \\
\hline Ácido 4 & $\nabla$ & Doce 4 & $\nabla$ & Ácido 1 & $\nabla$ & Controlo & $\nabla$ \\
\hline Ácido 3 & $\nabla$ & Salgado 3 & $\mathrm{X}$ amargo & Controlo & $\nabla$ & Salgado 2 & $\mathrm{X}$ amargo \\
\hline Doce 3 & $\nabla$ & Controlo & $\nabla$ & Salgado 1 & $\mathrm{X}$ amargo & Doce 1 & $\nabla$ \\
\hline Controlo & $\nabla$ & Amargo 3 & $\nabla$ & Amargo 2 & $\nabla$ & Ácido 1 & $\nabla$ \\
\hline Amargo 3 & $\nabla$ & Doce 3 & $\nabla$ & Amargo 1 & $\nabla$ & Salgado 1 & $\nabla$ \\
\hline Salgado 3 & $\mathrm{X}$ amargo & Ácido 3 & $\nabla$ & Doce 1 & $\nabla$ & Amargo 1 & $\nabla$ \\
\hline
\end{tabular}

Legenda: 1 - Maior concentração; 4 - Menor concentração; E - Lado esquerdo da língua; D - Lado direito da língua. Quando gosto alterado, é descrito qual o gosto identificado após o X. provas gustativas várias vezes por dia, juntamente com associação mental com a perceção sensorial. Durante todo o processo de recuperação, embora soubesse dos riscos cirúrgicos, a doente nunca pensou que a sua alteração do paladar pudesse ter um impacto tão grande na sua vida. E reconhece que, quando se depa-

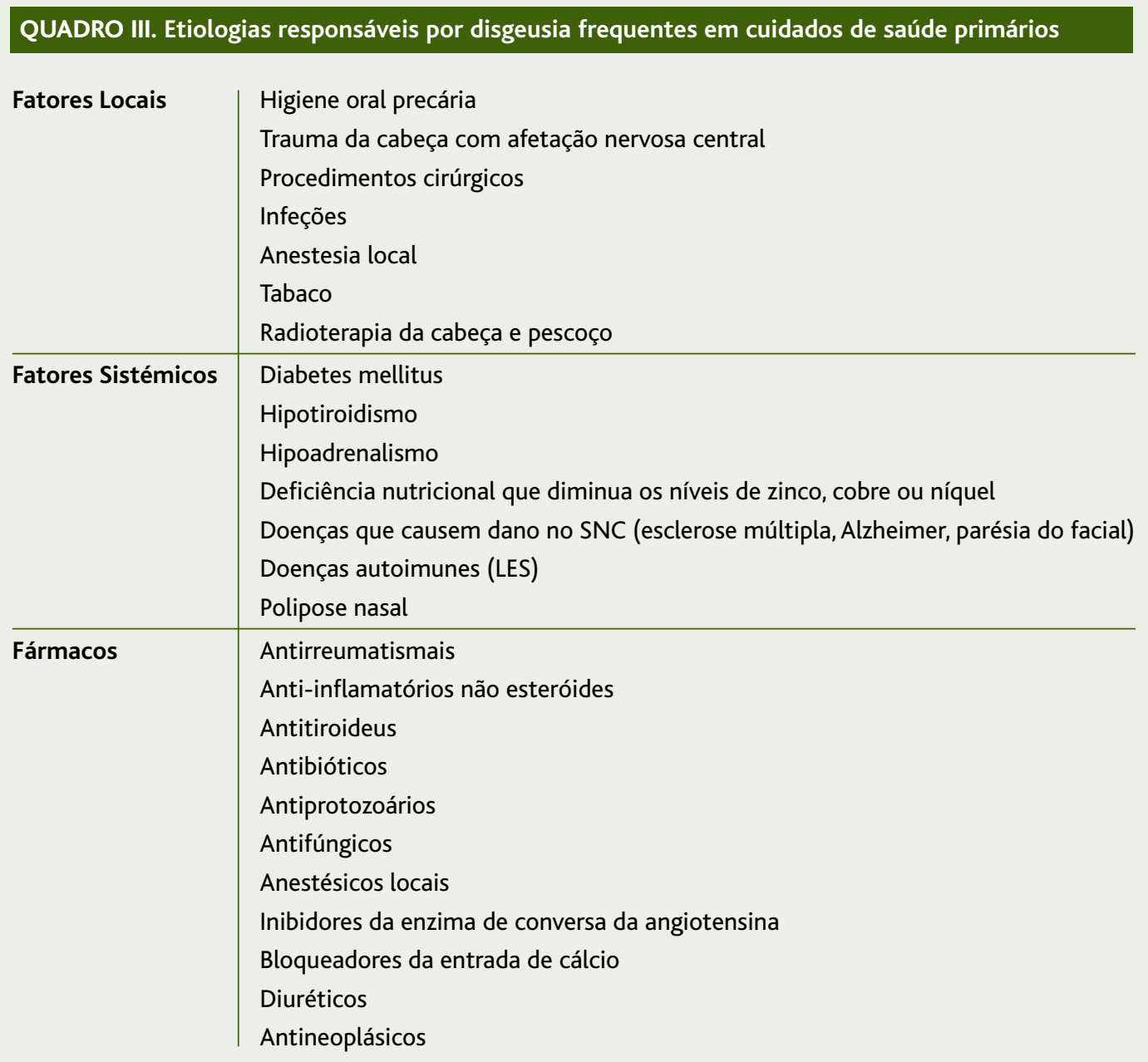


rou com esta dificuldade, foi difícil aceitar a sua condição, particularmente pelo facto de a mesma poder ser definitiva.

\section{COMENTÁRIO}

A digeusia é uma complicação rara da amigdalectomia, mas de elevada importância para o doente.

Muitos são os fatores que podem causar disgeusia num doente; podem ser fatores locais, sistémicos ou fármacos, como é representado no Quadro III.

O ramo lingual do nervo glossofaríngeo dista $2-4 \mathrm{~mm}$ do pólo inferior da amígdala palatina ${ }^{7}$ e, em $21,5 \%$ dos casos, este está fortemente aderente à cápsula amigdalina. ${ }^{3,7}$ Este é um dos responsáveis pela transmissão sensorial gustativa, juntamente com o nervo da corda do tímpano e grande petroso superficial. ${ }^{8}$

A amigdalectomia pode afetar este ramo, direta (laceração, descontinuação) ou indiretamente (dano térmico por cauterização, compressão), afetando a perceção gustativa do doente. ${ }^{4,7}$

Atualmente não há guidelines baseadas na evidência para o diagnóstico. No entanto, importa não esquecer que é importante esclarecer a causa da alteração do gosto, pois podemos estar perante uma doença sistémica potencialmente tratável ou precocemente diagnosticável. Devemos ter consciência de que o gosto alterado do doente influenciará a sua qualidade de vida e o seu dia-a-dia, podendo culminar em distúrbios alimentares/nutricionais e mesmo do foro psiquiátrico. ${ }^{3,5,7}$

Os casos de disgeusia pós amigdalectomia estão subreportados. Apresentam uma evolução relativamente rápida (disgeusia de curta duração) com recuperação completa em poucas semanas e sem tratamento. Há, no entanto, uma pequena percentagem de casos que mantém a sua alteração gustativa até dois anos após cirurgia. ${ }^{8}$ Este é um desses raros casos de disgeusia de longa duração.

Nos cuidados de saúde primários, as queixas de alterações gustativas são queixas vagas e sem relação aparentemente lógica com outras patologias, pelo que são facilmente negligenciadas na consulta. Este é um problema para o qual os médicos de família estão pouco alertados, dada a reduzida produção na literatura acer- ca deste tema, nomeadamente os relatos de caso. É importante a descrição destas situações. Atualmente não existem dados relativos à incidência/prevalência das alterações do paladar em cuidados de saúde primários em Portugal.

A maioria das alterações gustativas tem uma evolução benigna. No entanto, a avaliação do doente não deve ser descuidada, nem tão pouco desvalorizadas as suas queixas. ${ }^{8}$ Cabe-nos a nós estar atentos e ter um papel ativo na valorização, avaliação, orientação e tratamento das alterações do paladar, entre elas a disgeusia.

\section{REFERÊNCIAS BIBLIOGRÁFICAS}

1. Collet S, Eloy P, Rombaux P, Bertrand B. Taste disorders after tonsillectomy: case report and literature review. Ann Otol Rhinol Laryngol. 2005;114(3):233-6.

2. Landis BN, Welge-Luessen A, Brämerson A, Bende M, Mueller CA, Nor$\operatorname{din} \mathrm{S}$, et al. Taste Strips: a rapid, lateralized, gustatory bedside identification test based on impregnated filter papers. J Neurol. 2009;256(2): 242-8.

3. Tomofuji S, Sakagami S, Kushida K, Terada T, Mori H, Kakibuchi M. Taste disturbance after tonsillectomy and laryngomicrosurgery. Auris $\mathrm{Na}-$ sus Larynx. 2005;32(4):381-6.

4. Leong SC, Karkos PD, Papouliakos SM, Apostolidou MT. Unusual complications of tonsillectomy: a systematic review. Am J Otolaryngol. 2007;28(6):419-22.

5. Velmurugan SM. Dysgeusia: a review. Asian J Pharm Clin Res. 2013;6(4):16-8.

6. Mueller C, Kallert S, Renner B, Stiassny K, Temmel AF, Hummel T, et al. Quantitative assessment of gustatory function in a clinical context using impregnated "taste strips". Rhinology. 2003;41(1):2-6.

7. Uzun C, Adali MK, Karasalihoglu AR. Unusual complication of tonsillectomy: taste disturbance and the lingual branch of the glossopharyngeal nerve. J Laryngol Otol. 2003;117(4):314-7.

8. Malaty J, Malaty IA. Smell and taste disorders in primary care. Am Fam Physician. 2013;88(12):852-9.

\section{CONFLITO DE INTERESSES}

Os autores declaram não ter conflitos de interesses.

\section{ENDEREÇO PARA CORRESPONDÊNCIA}

Óscar de Barros

Rua Dr. Francisco Sá Carneiro, lote 2, n. ${ }^{\circ} 150,2{ }^{\circ}$ Fte

3000-194 Coimbra

E-mail: oscardcbarros@gmail.com

Recebido em 27-07-2014

Aceite para publicação em 02-06-2015

Artigo escrito ao abrigo do novo acordo ortográfico. 


\section{ABSTRACT}

\section{DYSGEUSIA: A CASE REPORT}

Introduction: The distortion of the sense of taste (dysgeusia), transient or permanent, may have significant impact on the lives of patients. It may be associated with weight loss, anorexia, malnutrition, depressive symptoms, and a significant reduction in quality of life. Dysgeusia can have several causes. One surgical cause is tonsillectomy.

Case description: A 38 year-old female patient, with dysgeusia is presented. She had a history of bilateral chronic tonsillitis and a cold blade tonsillectomy was performed with electrocautery of tonsillar hemorrhages without complications. After surgery she complained of dysgeusia characterized by lack of taste discrimination, stating that all foods seemed bitter. Six months after surgery, at a follow-up consultation, her gustatory function was evaluated with taste strips. This revealed changes in taste discrimination. She experienced a bitter taste, even when stimulated with sour, sweet or salty stimuli. The patient began daily gustatory rehabilitation, working on mental associations with sensory stimuli. She repeated the gustometry one year after surgery. The patient improved, although selective dysgeusia persisted with a bitter sensation in the presence of salt at low concentration.

Comment: Dysgeusia is a rare complication of tonsillectomy, but can have great impact on the lives of patients. The surgical procedure can damage sensory nerves responsible for taste, directly or indirectly. There are few therapeutic options. Cases are under reported in the literature, and tend to be benign. Dysgeusia can have various causes, such as antihypertensive drugs, poor oral hygiene, surgery, infection, smoking, diabetes, autoimmune diseases, anti-microbial agents, and anti-inflammatory drugs. In primary health care, taste complaints often have no obvious explanation at the initial presentation. In Portugal there is no data on their incidence. It is important to sensitize clinicians to this disease. Patients' complaints about changes in taste should be addressed because of the impact that it has on the quality of life and possible treatable causes. Patients with dysgeusia require assessment, advice and treatment.

Keywords: Dysgeusia; Taste; Tonsillectomy. 